\title{
A DEVELOPMENT OF WIRELESS CLASSROOM FRAMEWORK FOR CONSTRUCTION TECHNOLOGY COURSES
}

\author{
Chul S. Kim, Laura Lucas \\ Indiana University-Purdue University, Indianapolis
}

Introduction

Many courses in the Construction Technology (CNT) Program in Indiana UniversityPurdue University Indianapolis (IUPUI) are taught in traditional classrooms that are not equipped with computers. This is partially due to the lack of funding to provide enough computer facilities; however, it is more because computers are not currently deemed integral in the teaching and learning in these courses. Thanks to the rapid progress in information technology, classrooms are now changing. More instructional content is being delivered and received through a computer environment, electronic class notes on the Web, for example. Classroom activities such as discussion and testing now can be done online.

But even the non-computer-based construction courses could take advantage of using computers for various class activities. The immediate possibilities are to use computers to collect students' feedback and adjust the lecture based on the feedback while the class is in session: in other words, real-time assessment of the students and customized lectures based on the assessment. However, there still exists the problem of limited computer resources even if the necessity is justified. On the other hand, these courses still need to meet in non-lab classrooms to better facilitate discussions and multimedia presentations, or because of large class sizes.

The solution for both the limitation and the need is to use mobile computing devices in the classrooms. Mobile PCs (i.e. Pocket PCs, PDA) or laptop computers have rapidly become affordable yet are capable of computing at acceptable levels for most needs. A wireless network is readily available on the IUPUI campus which allows students to use mobile devices to connect to course content on the Internet. The technology for mobile computing now has reached the level of common availability so that it can be easily implemented in classrooms ${ }^{2}$. This research was performed to prepare a database framework for a wireless mobile PC based system that can administer quizzes and exams in construction course classrooms.

Goals and Objectives

The primary goal of this project is to enhance teaching and learning in construction technology at IUPUI by the increasing use of information technology tools for improving 
teaching and learning. The secondary goal is to establish an infrastructure of wireless classroom for construction technology education to provide a platform for wider applications.

The primary objective of this project is to develop a database framework of a system that can provide continuous and immediate feedback by adapting wireless classroom technology and implement it into more of the construction classrooms. By utilizing wireless classroom technologies, more interaction among the students and the instructor will be based on real-time assessment of the students in an otherwise lecture-based classroom.

Development of Wireless Classroom Framework

Various wireless classroom technologies are integrated and adapted to the course for the initial setup of the wireless construction classroom. This project requires two areas of development on the software side: a database system and an authoring system based on wireless technology. The database is a framework in which exam questions and student assessment data are stored.

Courses Selected for Adaptation and Implementation

This project selected two of the construction courses in the Construction Technology program at IUPUI to adapt the wireless technologies with plans to expand later into additional courses. The selected courses are: an introductory construction course titled Building Systems and Materials (ART 165) and a sophomore level course titled Quantity Survey (CNT 280). The courses have a great potential for adapting wireless technology and serve as good representatives for other department-wide courses because of their course contents and student populations.

The courses have been taught in a non-computer equipped classroom in conventional 'lecture and exam' settings. Besides a few sessions of guest lectures and field trips, most course content is delivered requiring minimal interaction of class participants. The materials presented in the classes heavily depend on graphics as well as plain text.

These courses cover quite extensive topics by dealing with properties, systems, and quantity survey of various construction materials such as concrete, steel, wood, and masonry. The emphasis often put on the breadth rather than on the depths of topics resulting in difficulties in tracking students' learning and understanding, in a formative manner.

Wireless Network Environment for Classroom

Indiana University-Purdue University Indianapolis (IUPUI) has a wireless network available throughout the campus to staff and students. Anyone with a valid university account can access the Internet and the university's Intranet through VPN (Virtual Private Network). Currently, any instructional materials can be accessed using this network service. However, there are some security concerns that have to be resolved before the wireless service can be used in classrooms. 
The researchers at Purdue University identified the concerns as follows; wireless devices can form a local network between students which can be abused as a pathway for cheating in exam; openness of wireless devices to the Internet can promote distraction to students during class; in certain cases, access to the course Website from a remote place other than in the classroom needs to be prohibited for the purpose of tracking attendance ${ }^{1}$. They propose the addition of a server to the existing network to prevent these problems listed above.

The network environment for this project must be flexible enough to accommodate various needs of instructor by allowing controlled access to the course materials. It also has to be scaleable for the changing requirement of the course as the system matures. The security and authentication issues are well recognized during the implementation as a leading problem to solve in the wireless classroom.

\section{System Configuration}

This project utilizes the Internet browser as a platform on which the lectures run. The World Wide Web related languages (HTML, Visual Basic Script, etc.) combined with interactive presentation software are used as the tools for authoring lectures.

The student assessment module references available technologies from existing works such as Oncourse ${ }^{4}$ and Wireless Quizzing ${ }^{3}$. The format of the Oncourse exams work well within the current desktop computer setting but was not suitable for many mobile devices, particularly Pocket PCs. Pocket PCs operate on much smaller screens than desktop computers which limits the size of page a Pocket PCs can view at once. It is thus desirable to have one question per page on the PocketPC screen where as Oncourse requires all questions to be on one page.

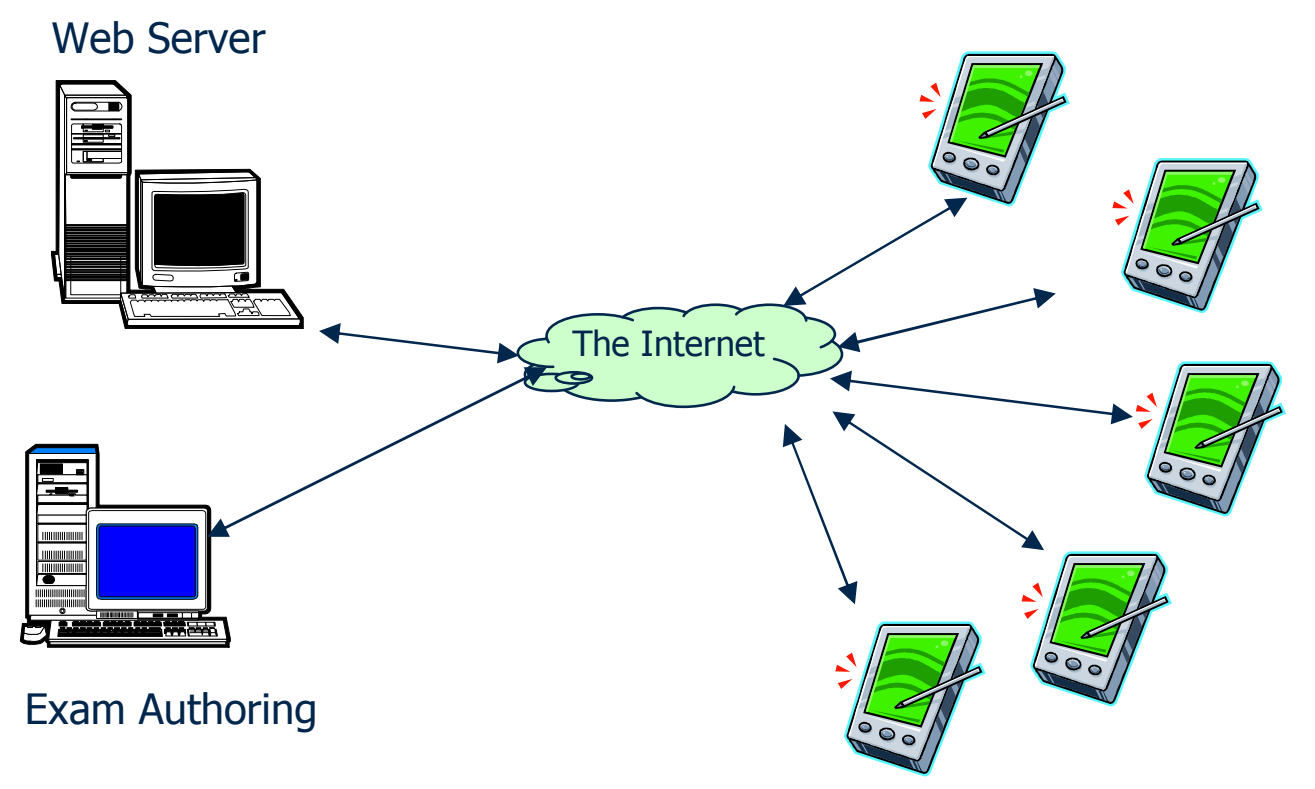

Student Units (Wireless Mobile PCs)

Figure 1 System Configuration 
On the other hand, Oncourse's exam authoring tools are very useful for putting together an exam easily. To incorporate a new exam format for the PDA while utilizing Oncourse's authoring tools, the export functionalities of Oncourse can be used to create intermediate exam source data. The format of the exported exam source data is in plain text with a proprietary hierarchy written as an ASP (Active Server Page) file.

In order to integrate the fragmented wireless technologies into the construction classroom, the infrastructure has to be developed. This project uses a generic database and software running on a small server computer which can be easily obtained as off-the-shelf products. The software runs in the web server which can be accessed by any web browsers.

As shown in Figure 1, student units access the course materials directly through the Internet using web browsers. Authoring is also done using a web browser through the Internet connection. The server running on Windows XP Professional ${ }^{\mathrm{TM}}$ operating system provides Internet Information Service (IIS) by publishing the data in the server on the web pages.

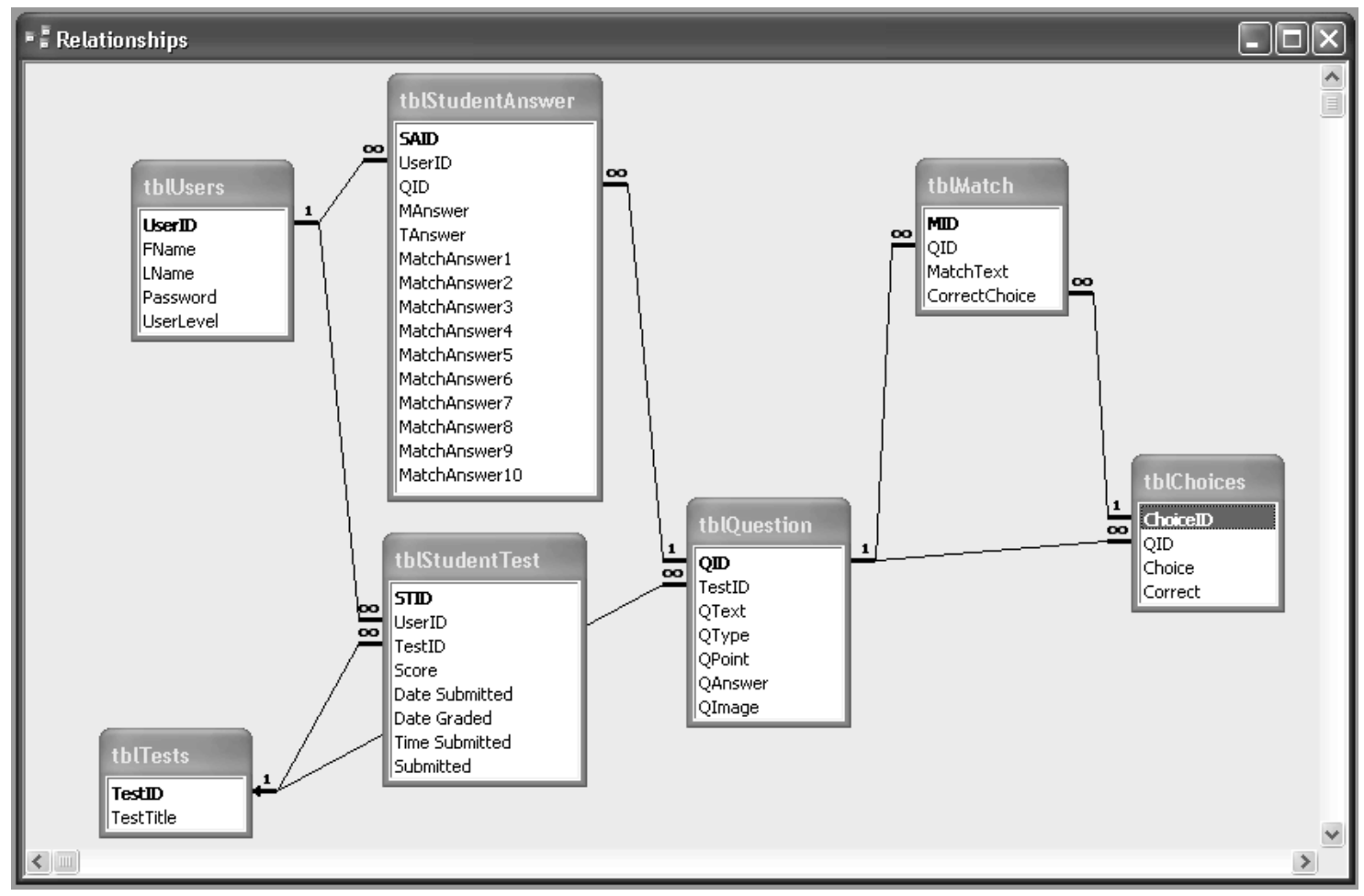

Figure 2 Schema Diagram of the Database

Database Framework

As shown in the schema diagram (layout of database entities) in Figure 2, the database consists of seven tables: Users, Tests, StudentTest, Question, Match, Choices, and StudentAnswer.

- Users Table (tblUsers): tblUser stores user information such as names, user ID, and password. The user level is determined during the authentication process by 
screening the user ID and user level. The administrators can access exam authoring pages and students' answers and grades while the regular users are limited only to the exam taking pages.

- Tests Table (tblTests): tblTests stores test ID and title.

- Student Test Table (tblStudentTest): tblStudentTest joins tblUser and tblTests to store incidents of exams taken by students. This table also stores the timestamps of exams and grading as well as exam scores.

- Question Table (tblQuestion): tblQuestion contains questions, question types (i.e. multiple choice, matching), points, and links to the images.

- Choices Table (tblChoice): tblChoice joins tblQuestion by listing choices of the question. It also contains correct answer of the question.

- Match Table (tblMatch): tblMatch stores match choices when the question type is "match."

- tblStudentAnswer (tblStudentAnswer): tblStudentAnswer stores answers entered by students while taking exam.

User Interfaces

The user interface for the students was designed with the ease of use as the system's first priority. It uses standard web browser controls such as text input box, buttons, and hypertext links to enter input and navigate through the web pages. The number of pages a student has to access in order to complete an exam is six. The last page is to display the current status of student's answer.
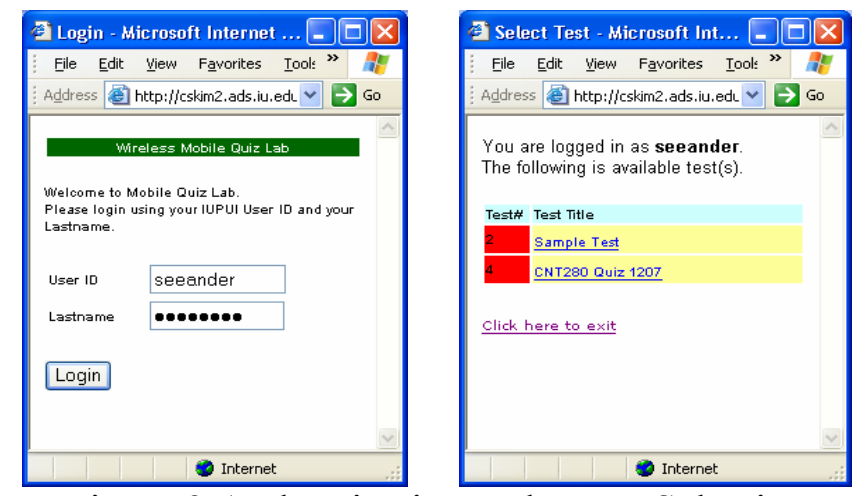

Figure 3 Authentication and Exam Selection

As shown in Figure 3 the first two pages are for authentication and exam selection. In the authentication page, a user enters user ID and a password. Depending on the user level designation, a user will be directed to the administration page or the exam list page. The second picture in Figure 3 shows the list of exams that are currently available to the student logged in. By clicking on the links, the student is led to the question list page which is shown in Figure 4.

Figure 4 also depicts two types of questions, multiple choice and match question. For multiple choice questions, the student selects a choice as an answer and submits it. Match questions are answered using pull-down boxes with which students make a selection as a match to each question. 

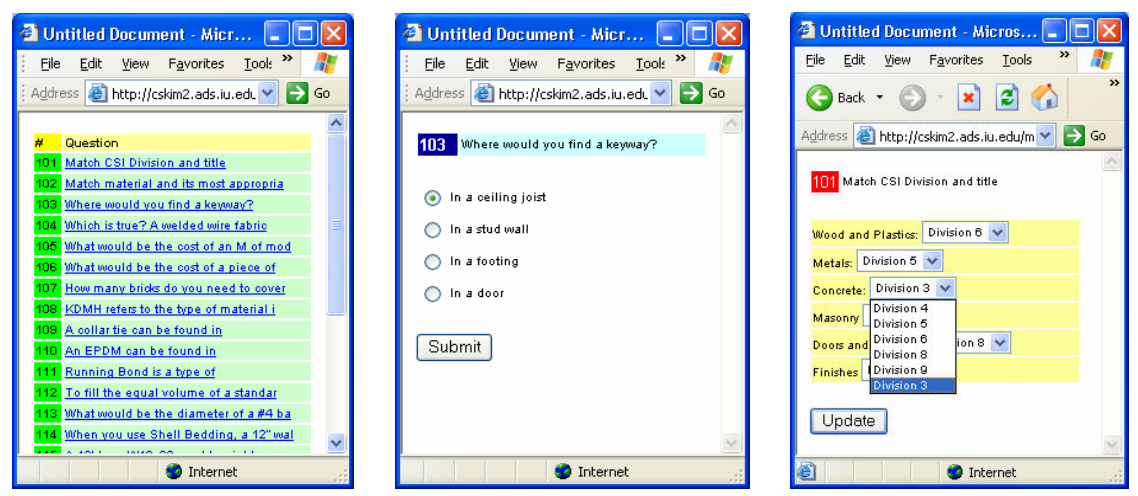

Figure 4 Question List, Multiple Choice Question and Match Question

Because each question is answered singly on each page, it is not easy for the students to keep track of questions that are answered and that are not in case they do not proceed in order. An additional page is provided to display the current status of the student's advancement in answering question as shown in Figure 5. This page shows the list of questions and indicates the ones that are not answered by the student so that no questions are left unanswered.

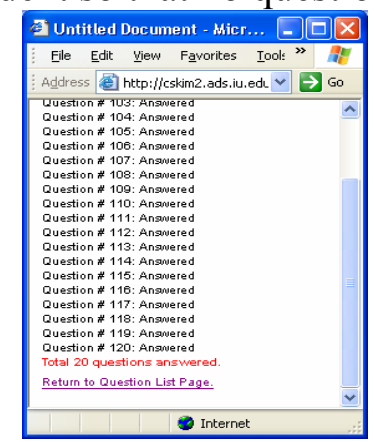

Figure 5 The Status of Student Answers

Pilot Test and Assessment

After the system was developed, it was pilot tested in CNT 280 (Quantity Survey) class. The number of students was 20 and the number of questions was 50. Because only 10 Pocket PCs were available for the session, so the students were divided into two groups of 10 each. A two page instruction was provided to each student along with a 5 minute tutorial. While the first group was taking the exam, the other was working on another class task. Once the first group finished, they switched the activities. Since the students were already familiar with general web page operation, no one had problem using the system. The times each student consumed taking the test was between 11 and 17 minutes.

Student interviews were conducted immediately after the exam in the form of group discussions. The reviews of the students were favorable over traditional paper format exams as well as the existing web-based quiz system available on campus as expressed during the student interview. The following is the list of the issues that came up during the development and the pilot test: 
- Hardware Maintenance: Several units of mobile PCs must be well maintained. The batteries should be fully charged and the units should be properly transported to classrooms. A cart with electrical outlets worked well for transporting, however, additional safety devices to keep the units from theft and abuse are necessary.

- Network Connection: Some units had difficulties in making connection to the network. About 10 minutes before each session was spent to ensure that the network connection was properly established.

- Screen Size: The size of screen is limited at 240 x 263 pixels. Although a user can scroll if the content is larger than the screen, it is desirable to design the contents to fit within the screen to minimize scrolling.

- Text Size: There are 5 different text sizes to choose from within Internet Explorer of mobile PCs. The default size was set to 'Medium' which was well accepted by the students. However, if the student changes text size, the page layout may be unorganized.

Summary

In order to monitor student learning in non-computerized classrooms, a mobile PC-based wireless exam system was developed. Using web-based interfaces running on a database, students can take quizzes and exams to provide immediate feedback to the instructor. After a few pilot tests, the system was found acceptable by the students. There also were some reservations in terms of hardware maintenance and software interface which can be overcome with fine tuning of hardware and software.

The primary outcome of this work is the development of the database framework. Upon this framework, many additional functionality and features can be built to meet specific classroom, content, or instructor needs. The framework also has a potential for expanded application to other field of engineering and technology education than construction.

Through this work, it was found that bringing the computer to the existing classroom was often more efficient and economical way to improve the collection of formative data on student learning. Faculty could use the immediate feedback from in-class quizzes or survey to redirect classroom discussion or activities to concentrate on students' needs. This just-in-time aspect of teaching is proven to greatly enhance learning.

\section{Reference}

(1) Adewunmi, A., et. Al (2003), Enhancing the In-classroom Teaching/Learning Experience Using Wireless Technology. The $33^{\text {rd }}$ ASEE/IEEE Frontier in Education Conference, IEEE, Boulder, Colorado

(2) Alford, K. L. and Hill, J. M. D. (2003), Adding PDAs to Your Teaching Toolkit. The 33 ${ }^{\text {rd }}$ ASEE/IEEE Frontier in Education Conference, IEEE, Boulder, Colorado

(3) Homan, S. R., \& Wood, K. (2003). Taming the Mega-Lecture: Wireless Quizzing: Campus Technology. Retrieved November 21, 2003, from http://www.campus-technology.com/campusmobility/article.asp?id=8251 
(4) Indiana University. (2003). The Oncourse Story. Retrieved December 10, 2004 from

http://oncourse.iu.edu/news/story.html

\section{Author Biography}

Dr. CHUL KIM is an assistant professor in the Department of Construction Technology in Indiana University-Purdue University, Indianapolis. He earned his M.S. and Ph.D. in Civil Engineering from University of Illinois at Champaign-Urbana. His technical research is in application of information technology (IT) in construction project management and in construction classrooms.

Ms. LAURA LUCAS is a lecturer in the Department of Construction Technology in Indiana UniversityPurdue University, Indianapolis. She earned B.Arch. from Ball State University and MBA from Indiana University. She is specialized in collecting and analyzing assessment data and curriculum development in response to assessment data. 